\title{
Analysis of the non-parallel flow-based Green's function in the acoustic analogy for complex axisymmetric jets
}

\author{
M. Z. Afsar* \\ Department of Mechanical \& Aerospace Engineering, University of Strathclyde, Glasgow, G1 I XJ, UK.
}

A. $\operatorname{Sescu}^{\dagger}$

Department of Aerospace Engineering, Mississippi State University, Starkville, MS, USA.

V. Gryazev $\ddagger$

School of Engineering and Material Science, Queen Mary University of London, Mile End Road, London, E1 4NS, UK.

A. Markesteijn $\S$

School of Engineering and Material Science, Queen Mary University of London, Mile End Road, London, E1 4NS, UK.

S. Karabasov II

School of Engineering and Material Science, Queen Mary University of London, Mile End Road, London, E1 4NS, UK.

This paper considers how a complex axisymmetric jet modifies the structure of the propagator tensor in Goldstein's generalized acoustic analogy. The jet flow we consider is in general a dual stream flow that operates either as a single jet or a complex co-axial jet flow. The latter of which is of interest to turbofan engine manufacturers. The form of the acoustic analogy that we use here is based on our recent work on jet noise modeling (Afsar et al. 2019, PhilTrans. A., vol. 377) that highlighted the importance of non-parallel flow effects in the correct calculation of the propagator. The propagator calculation takes advantage of the fact that mean flow non-parallelism enters the lowest order asymptotic expansion of the former at sufficiently low frequencies of the same order as the jet spread rate. Whilst this might seem restrictive, our previously reported calculations at high subsonic and mildly supersonic jets indicate that the subsequent jet noise predictions remain accurate up to the peak frequency (typically at a Strouhal number based on jet velocity and diameter of $\approx 0.5-0.6$ ) for the small angle acoustic radiation. One of critical assumptions of this approach is that the mean flow speed of sound squared is given by either the Crocco relation (in unheated jets) or the Crocco-Busemann relation for heated flows. Our analysis for the dual stream complex axisymmetric jet however shows that the latter assumption (in the form of Crocco-Busemann formula) is no longer an accurate representation of the speed of sound variation. We therefore present a more general form of the asymptotic analysis than that used in Afsar et al. (2019a \& b). For the complex jet mean flow field, the mean flow speed of sound is otherwise arbitrary but must remain a single-valued function of the streamwise mean flow. The predictions based on this approach are shown to remain accurate up to the peak frequency. We discuss how to extend the range of validity by utilizing a suitable composite asymptotic solution for the Green's function problem.

\section{Introduction}

$\mathrm{M}$ $\mathrm{UCH}$ of the recent progress in our understanding of the mathematical structure of jet noise and its modeling within a prediction model is based, to a large extent, on Lighthill's acoustic analogy approach [1] and its variants. In particular, in the last decade or so, this has been in the form of Goldstein's generalized acoustic analogy [2]. The latter has provided an ab-initio platform to consider both the wave propagation (which enters via a solution to the adjoint

\footnotetext{
*Chancellor's Fellow. AIAA Member.

$\dagger$ Associate Professor, and AIAA Associate Fellow.

† PhD Candidate. AIAA Member.

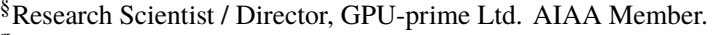

ITReader in Computational Modelling. AIAA Senior Member.
} 


$\begin{array}{lllll}\text { Operating Point (OP) } & \text { Description } & M_{J} & T R & M a \\ \text { OP 1.3 } & \text { Dual stream heated ideally expanded } & 0.86 & 2.7 & 1.4 \\ \text { OP 1.7 } & \text { Single stream unheated subsonic } & 0.64 & 1.0 & 0.64\end{array}$

Table 1 Gryazev et al (2019) [6] test cases

linearized Euler equations - referred to here as the ALEE) and turbulence modeling for the generalized auto-covariance tensor. The latter reduces to the ordinary Reynolds stress auto-covariance tensor, which even prior to serious efforts in jet noise modeling was measured in experiments on high-order turbulence correlations and structure functions albeit in more homogeneous turbulent flows [3]. In the present paper we use an asymptotic theory to calculate the propagator tensor in the generalized analogy [4]. The asymptotic theory takes advantage of the dominant balance between the temporal frequency and jet spread rate as being the distinguished limit where non-parallel flow effects enter the lowest order expansion of the ALEE. The predictive capability of the theory was analyzed in our two recent papers (Afsar $e t$ al. [4] \& [5]) for axisymmetric jets having high subsonic acoustic Mach number $\left(M a=U_{J} / c_{\infty}\right)$ as well as for mild supersonic heated and unheated jets. In this paper, however, we focus on testing this approach for the more industrially realistic scenario of a dual-stream high speed co-axial jet that operates in both heated and unheated conditions. See $\operatorname{table} 2$ for a summary of the operating points that we consider in this paper.

\section{Technical complications associated with dual-stream mixing}

The Reynolds averaged Navier-Stokes (RANS) mean flow field and Large-eddy Simulation (LES) database for both cases were reported in [6]. Since both streams in the Gryazev et al. numerical simulations emerge from round nozzles (see Fig. 1) and are, therefore, axisymmetric, the basic form of the inner equations in $(Y, r)$ co-ordinates derived by Goldstein et al. ([7], Eqs. 5.18-5.20) continue to hold. Here, $Y=\epsilon y_{1}=O(1)$ is the slow streamwise length and $r=O(1)$ is an arbitrary radial distance. Both the jet mean flow and the vector Green's function solution of the ALEE evolve under these scales. Goldstein et al. showed that the inner equations reduce to a hyperbolic partial differential equation (PDE) under the one-to-one transformation of independent variable $(Y, r) \rightarrow(Y, U)$ where the frequency is re-scaled to $\Omega=\omega / \epsilon=O(1)$. This final equation that they found (Eq. 5.31 in their paper) was re-derived in a much simpler manner in Afsar et al. [4] \& [5]. It was also extended to arbitrary heated flows possessing $O(1)$ static jet temperature ratio, $T R=T_{J} / T_{\infty}$. In both latter references it was shown that the transformation of variables can be performed prior to asymptotic analysis inasmuch as mapping $\left(y_{1}, r\right) \rightarrow\left(y_{1}, U\right)$ in which the Favre-averaged mean flow speed of sound, $\widetilde{c^{2}}$ satisfied either the Crocco relation (unheated jet) or the Crocco-Busemann relation (heated case).

In the present paper, however, we show that for the complex axisymmetric flow problem shown in Fig. [?? the interaction between both streams introduce a number of complications in terms of the physics of the problem but also in terms of the subsequent reduction of the inner asymptotic ALEE equations at $\Omega=O(1)$ frequencies.

One of the main complications is that $V_{r}$ can be sizeable in regions of the flow along the shear layer and where both streams interact. The latter effect is to introduce apparent well-resolved "bumpiness" in the steamwise component of the mean flow advection vector, $\bar{X}_{1}=D U / D \tau \equiv \tilde{\boldsymbol{v}} \cdot \boldsymbol{\nabla} U=V_{r} \partial U / \partial r$. See Figs. 2 and 3. More importantly, the latter interaction between the initial shear layers and the fully mixed zones causes a wide departure from the Crocco-Busemann relation for OP1.3. This is shown in Figs. 4 and 5.

In the remaining part of the paper we firstly summarize the generalized acoustic analogy equations. We then extend the analysis in references [4] \& [5] to consider flows where the $\widetilde{c^{2}}=f\left(U\left(y_{1}, r\right)\right)$ but is otherwise arbitrary (i.e. is not given by either Crocco or Crocco-Busemann relations). Our aim is to complete the Green's function calculations for the conference and present initial noise predictions for the jet conditions shown in Table 2 . These conditions correspond to the EU-funded Computation of Coaxial Jet Noise (CoJeN) project for which measured acoustic data exists.

\section{Generalized acoustic analogy}

Consider a region of non-homogeneous turbulence bounded within a high speed jet of order-1 acoustic Mach number, $M a=U_{J} / c_{\infty}$ and order-1 temperature ratio, $T R$. Pressure fluctuations within the jet propagate to the far field where they are perceived as sound. We use Goldstein's generalized acoustic analogy [2] to represent this process in a manner whereby the wave propagation is calculated via a propagator tensor that depends on ALEE solution and the acoustic spectrum depends on this and the Reynolds stress auto-covariance tensor, the latter of which is modeled appropriately 


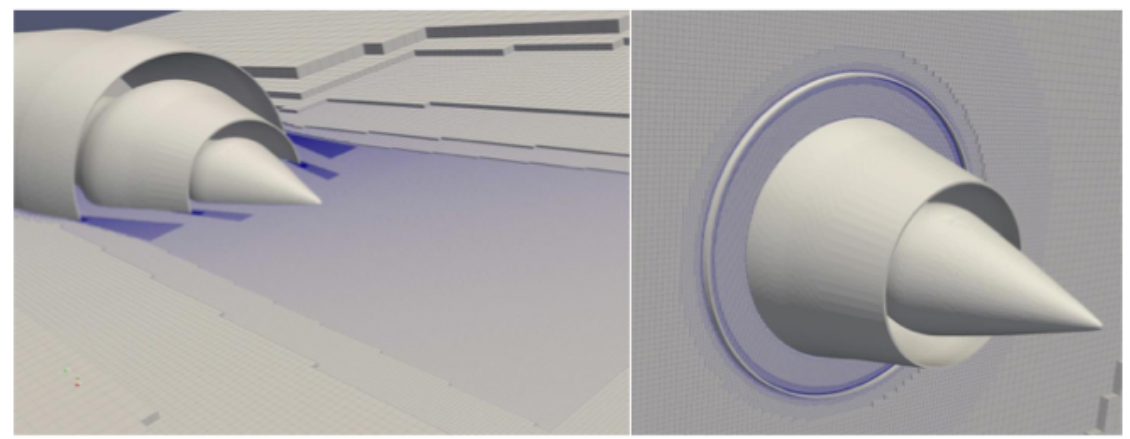

Fig. 1 Dual stream structure of flow. Three-dimensional view of the LES grid (reproduced from Fig. 1 in [6]

(see reference [5]). Let the pressure $p$, density $\rho$, enthalpy $h$, and speed of sound $c$ satisfy the ideal gas law equation of state $p=\rho c^{2} / \gamma$ and $h=c^{2} /(\gamma-1)$, where $\gamma$ denotes the ratio of specific heats.

The acoustic spectrum at the observation point, $\boldsymbol{x}=\left(x_{1}, \boldsymbol{x}_{T}\right)=\left(x_{1}, x_{2}, x_{3}\right)$, given by the Fourier transform

$$
I(\boldsymbol{x}, \omega) \equiv \frac{1}{2 \pi} \int_{-\infty}^{\infty} e^{i \omega \tau} \overline{p^{\prime}(\boldsymbol{x}, t) p^{\prime}(\boldsymbol{x}, t+\tau)} d \tau
$$

of the far-field pressure auto-covariance, $\overline{p^{\prime}(\boldsymbol{x}, t) p^{\prime}(\boldsymbol{x}, t+\tau)}$, can be expressed as a volume integral over a unit volume of turbulence at $\boldsymbol{y}=\left(y_{1}, \boldsymbol{y}_{T}\right)=\left(y_{1}, y_{2}, y_{3}\right)$ in the jet via

$$
I(\boldsymbol{x} ; \omega)=\int_{V_{\infty}(\boldsymbol{y})} I(\boldsymbol{x}, \boldsymbol{y} ; \omega) d \boldsymbol{y}
$$

where, $V_{\infty}(\boldsymbol{y})$ is the entire source region.

The pressure fluctuation in $(1)$ is defined as $p^{\prime}(\boldsymbol{y}, \tau) \equiv p(\boldsymbol{y}, \tau)-\bar{p}(\boldsymbol{y})$ where over-bars are denote time average,

$$
\bar{\bullet}(\boldsymbol{x}) \equiv \lim _{T \rightarrow \infty} \frac{1}{2 T} \int_{-T}^{T} \bullet(\boldsymbol{x}, t) d t
$$

such that $\bullet$ in $(3)$ is a place holder for any fluid mechanical variable.

Goldstein \& Leib ([8]; hereafter referred to as G \& L) showed that $I(\boldsymbol{x}, \boldsymbol{y} ; \omega)$ on right side of $[2]$ is given by formula,

$$
I(\boldsymbol{x}, \boldsymbol{y} ; \omega)=(2 \pi)^{2} \Gamma_{\lambda, j}(\boldsymbol{y} \mid \boldsymbol{x} ; \omega) \int_{V_{\infty}(\boldsymbol{\eta})} \Gamma_{\mu, l}^{*}(\boldsymbol{y}+\boldsymbol{\eta} \mid \boldsymbol{x} ; \omega) \mathcal{H}_{\lambda j \mu l}(\boldsymbol{y}, \boldsymbol{\eta} ; \omega) d \boldsymbol{\eta}
$$

Here, asterisks denote complex conjugate and the Einstein summation convention is being used with the Greek tensor suffixes ranging from $(\lambda, \mu)=(1,2,3,4)$ and the Latin suffixes from $(i, j, k, l)=(1,2,3)$. The ALEE (defined below in 7 s show that the Greens's function that enters (4) depends on the Favre-averaged mean flow field of the jet, 


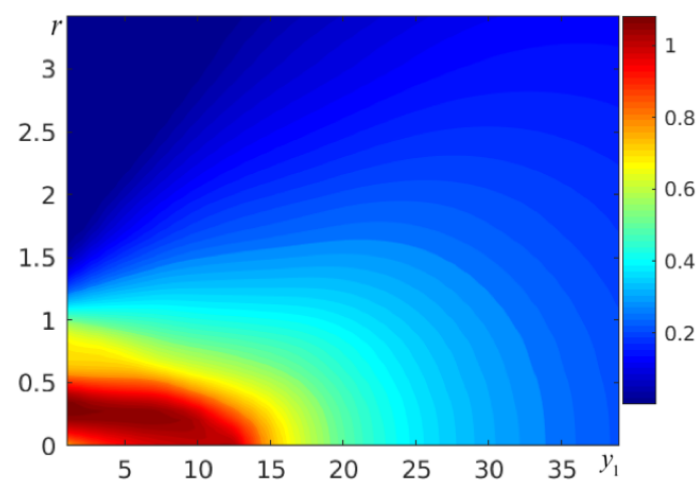

(a) Streamwise mean flow, $U\left(y_{1}, r\right)$

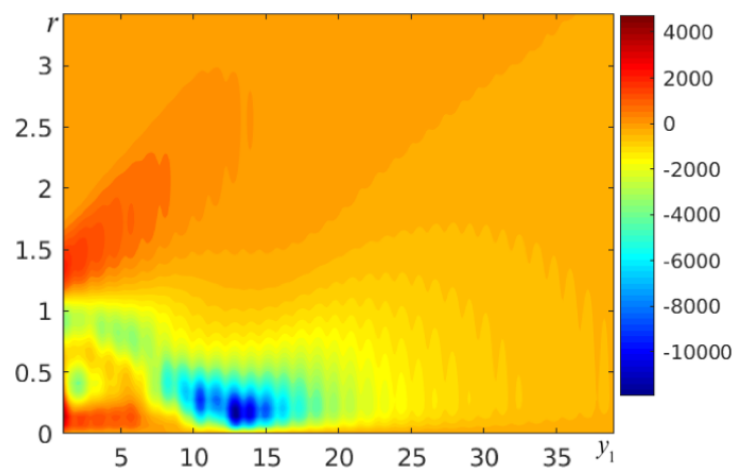

(c) Stremwise mean flow advection, $\bar{X}_{1}\left(y_{1}, r\right)$

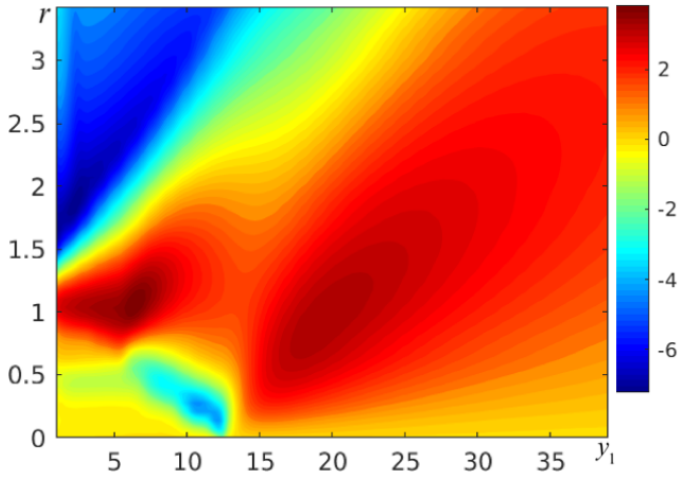

(b) Radial mean flow, $V_{r}\left(y_{1}, r\right)$

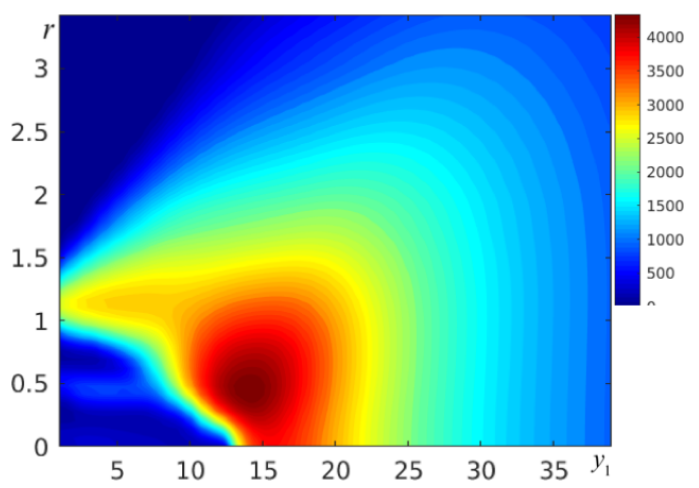

(d) Turbulent kinetic energy, $\bar{\rho} k\left(y_{1}, r\right)$

Fig. 2 Mean flow structure for OP1.3 in Table 2 .

$\tilde{\boldsymbol{v}}=\left\{\tilde{v}_{1}, \tilde{v}_{2}, \tilde{v}_{3}\right\}=\overline{\rho \boldsymbol{v}} / \bar{\rho}$ and mean speed of sound, $\widetilde{c^{2}}=\gamma \bar{p} / \bar{\rho}$, by the Fourier transform of the propagator tensor

$$
\Gamma_{\lambda, j}(\boldsymbol{y} \mid \boldsymbol{x} ; \omega) \equiv \Lambda_{\lambda \sigma, j}(\boldsymbol{y}) G_{\sigma}(\boldsymbol{y} \mid \boldsymbol{x} ; \omega):=\left(\delta_{\lambda \sigma} \frac{\partial}{\partial y_{j}}-(\gamma-1) \delta_{4 \sigma} \frac{\partial \tilde{v}_{\lambda}}{\partial y_{j}}\right) G_{\sigma}(\boldsymbol{y} \mid \boldsymbol{x} ; \omega)
$$

that involves an inner tensor product in suffix $\sigma$, of operator $\Lambda_{\lambda \sigma, j}(\boldsymbol{y})$, that spans $(4 \times 4 \times 3)$ dimensions corresponding to suffixes $(\lambda, \sigma, j)$ where comma after $j$ indicates that this suffix belongs to a derivative, and the first four components of the Fourier transform

$$
G_{\sigma}(\boldsymbol{y} \mid \boldsymbol{x} ; \omega)=\frac{1}{2 \pi} \int_{-\infty}^{\infty} e^{i \omega(t-\tau)} g_{\sigma 4}^{a}(\boldsymbol{y}, t-\tau \mid \boldsymbol{x}) d(t-\tau),
$$

of the five-dimensional adjoint vector Green's function, $g_{\sigma 4}^{a}(\boldsymbol{y}, \tau \mid \boldsymbol{x}, t)$, that appears on the left hand sides of the five ALEE (momentum, energy \& mass continuity) that were given previously in (4.8)-(4.10) of G\&L. The pressure-like Green's function component of $g_{\sigma 4}^{a}$ is subject to the strict causality condition $g_{44}^{a}(\boldsymbol{y}, t-\tau \mid \boldsymbol{x})=0$ for $t<\tau$ when $|\boldsymbol{x}| \rightarrow \infty$. The unit tensor in (5) is now a 4-dimensional Kronecker delta function and $G_{\sigma}(\boldsymbol{y} \mid \boldsymbol{x} ; \omega)$ is determined by the ALEE:

$$
\begin{aligned}
-D_{0} G_{i}+G_{j} \frac{\partial \tilde{v}_{j}}{\partial y_{i}}-\widetilde{c^{2}} \frac{\partial G_{4}}{\partial y_{i}}+(\gamma-1) \tilde{X}_{i} G_{4}-\frac{\partial G_{5}}{\partial y_{i}} & =0 \\
-D_{0} G_{4}-\frac{\partial G_{i}}{\partial y_{i}}+(\gamma-1) G_{4} \frac{\partial \tilde{v}_{i}}{\partial y_{i}} & =\frac{\delta(\boldsymbol{x}-\boldsymbol{y})}{2 \pi} \\
-D_{0} G_{5}+\tilde{X}_{i} G_{i} & =0
\end{aligned}
$$




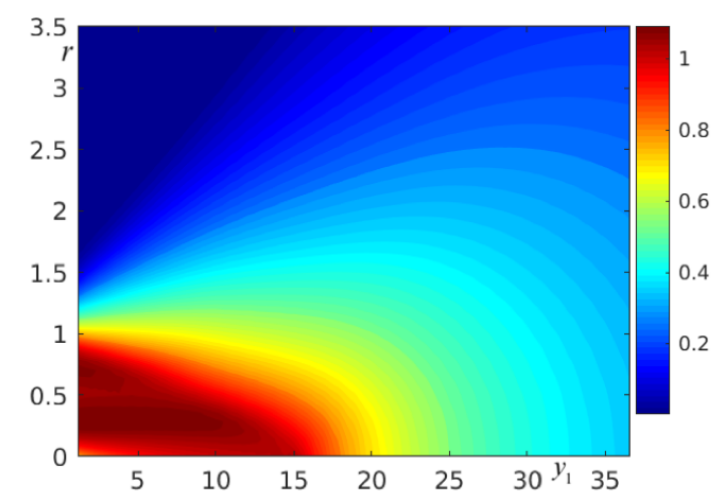

(a) Streamwise mean flow, $U\left(y_{1}, r\right)$

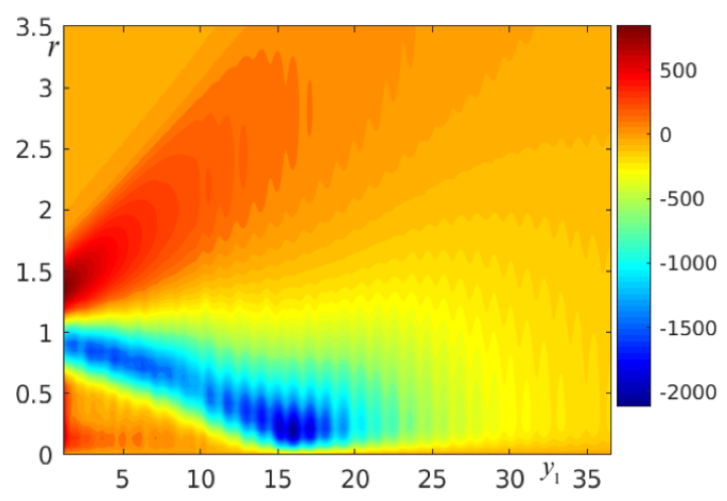

(c) Stremwise mean flow advection, $\bar{X}_{1}\left(y_{1}, r\right)$

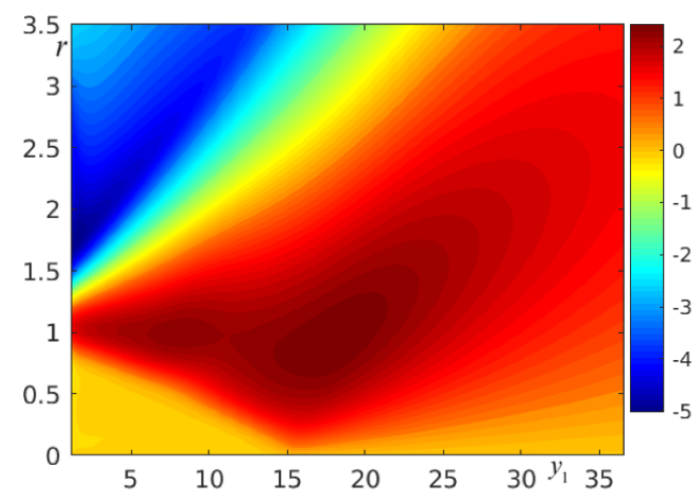

(b) Radial mean flow, $V_{r}\left(y_{1}, r\right)$

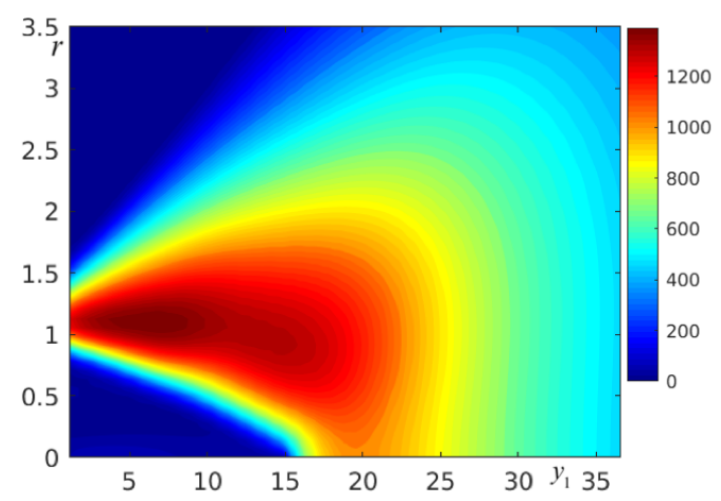

(d) Turbulent kinetic energy, $\bar{\rho} k\left(y_{1}, r\right)$

Fig. 3 Mean flow structure for OP1.7 in Table2.

where $D_{0} \equiv i \omega+\tilde{\boldsymbol{v}}(\boldsymbol{y}) \cdot \boldsymbol{\nabla}$ is the convective derivative and $\boldsymbol{\nabla} \equiv\left\{\partial / \partial y_{1}, \partial / \partial y_{2}, \partial / \partial y_{3}\right\}$ is the three-dimensional gradient operator. The coefficients in $(7)$ depend on the mean flow field through $\tilde{v}_{i}=\left(\tilde{v}_{1}, \tilde{v}_{2}, \tilde{v}_{3}\right) ; \tilde{c}^{2}(\boldsymbol{y}) \equiv \gamma \bar{p} / \bar{\rho}$, the mean flow speed of sound squared, and

$$
\tilde{\boldsymbol{X}}(\boldsymbol{y})=(\tilde{\boldsymbol{v}} \cdot \boldsymbol{\nabla}) \tilde{\boldsymbol{v}},
$$

the mean flow advection vector.

The tensor, $\mathcal{H}_{\lambda j \mu l}(\boldsymbol{y}, \boldsymbol{\eta} ; \omega)$ in (4) is related to the Fourier transform of the generalized auto-covariance tensor, $R_{\lambda j \mu l}(\boldsymbol{y}, \boldsymbol{\eta} ; \tau)$, of the stationary random function, $e_{\lambda j}(\boldsymbol{y}, \tau)=-\left[\rho v_{\lambda}^{\prime} v_{j}^{\prime}-\overline{\rho v_{\lambda}^{\prime} v_{j}^{\prime}}\right](\boldsymbol{y}, \tau)$, by the linear transformation $\mathcal{H}_{\lambda j \mu l}(\boldsymbol{y}, \boldsymbol{\eta} ; \omega):=\epsilon_{\lambda j \sigma m} H_{\sigma m \gamma n}(\boldsymbol{y}, \boldsymbol{\eta} ; \omega) \epsilon_{\mu l \gamma n}$ where $\epsilon_{\lambda j \sigma m} \equiv \delta_{\lambda \sigma} \delta_{j m}-\delta_{\lambda j} \delta_{\sigma m}(\gamma-1) / 2$ (see (5.12) to (5.13) in G \& L and Eqs. $2.9 \& 2.10$ in ASL19; note missing negative sign in the definition of $e_{\lambda j}$ below 2.10 in ASL19). The suffix ' 4 ' indicates enthalpy fluctuation via $v_{4}^{\prime}:=(\gamma-1)\left(h^{\prime}+v^{\prime 2} / 2\right) \equiv\left(c^{2}\right)^{\prime}+(\gamma-1) v^{\prime 2} / 2$ where $h^{\prime}$ is the fluctuating static enthalpy and $\left(c^{2}\right)^{\prime}$ is the fluctuations in the sound speed squared such that $v_{4}^{\prime} /(\gamma-1)$ denotes the moving frame stagnation enthalpy fluctuation [2].

The acoustic spectrum integral (2) is evaluated in cylindrical polar coordinates $\boldsymbol{y}=\left(y_{1}, r, \psi\right)$ with respect to an origin at the nozzle exit plane. Note this is commensurate with an axi-symmetric round jet for which we shall apply the new composite Green's function formula. Hence the mean flow field, commensurate with an axisymmetric jet, has components, $\boldsymbol{v}=\left(U, V_{r}\right)$. Moreover, if we let $\left(\boldsymbol{e}_{1}, \boldsymbol{e}_{r}, \boldsymbol{e}_{\phi}\right)$ be an orthogonal set of basis vectors in the cylindrical co-ordinate space, $\boldsymbol{G}=\left(G_{1}, G_{r}, G_{\phi}\right)$ in $\left(7 \mathrm{a}\right.$-c) can be expressed as a linear function of that basis by $\left(G_{i} \boldsymbol{e}_{i}\right) \boldsymbol{e}_{j}=G_{1} \delta_{j 1}+G_{r} \delta_{j r}+G_{\phi} \delta_{j \phi}$ where $\boldsymbol{G}=\left(G_{1}, G_{r}, G_{\phi}\right)$ are its respective components of $\boldsymbol{G}$ in the basis $\left(\boldsymbol{e}_{1}, \boldsymbol{e}_{r}, \boldsymbol{e}_{\phi}\right)$. 


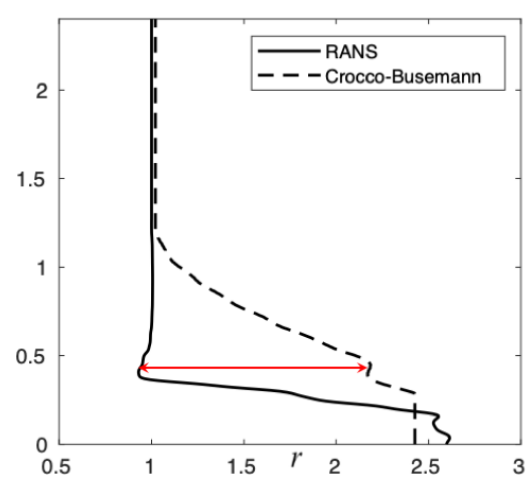

(a) $y_{1}=2$

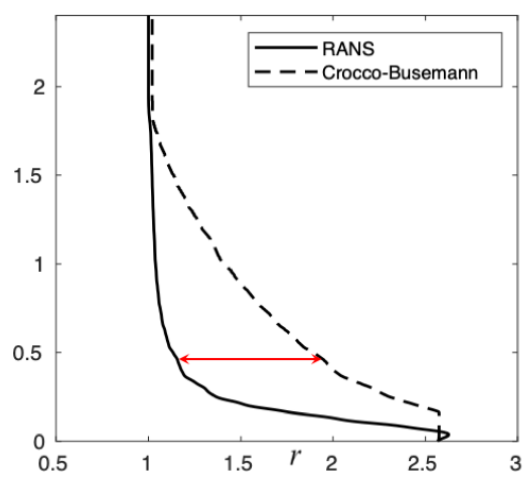

(c) $y_{1}=6$

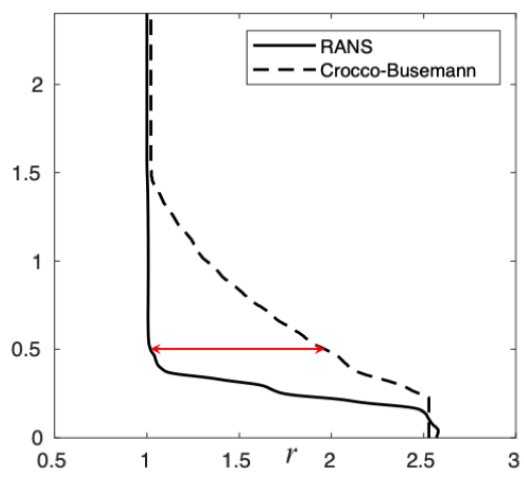

(b) $y_{1}=4$

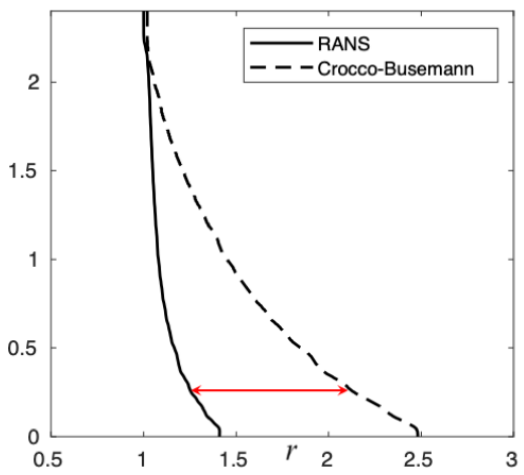

(d) $y_{1}=8$

Fig. 4 Comparison of the Crocco-Busemann (Eq. 17 in [5]) to the RANS mean flow $\widetilde{c^{2}} / c_{\infty}^{2}$ for OP1.3 at various points in the jet.

\section{Transformation of ALEE for jets with arbitrary mean speed of sound, $\widetilde{c^{2}}$}

Before we perform any asymptotic analysis to determine the composite Green's function, we transform the independent and dependent variables in (7) by taking $U$ to be one of the independent variables of choice; i.e. under the one-to-one mapping $\left(y_{1}, r\right) \rightarrow\left(y_{1}, U\right)$ where $r \equiv\left|\boldsymbol{y}_{T}\right|=\sqrt{y_{2}^{2}+y_{3}^{2}}$ (see [7] \& [5]). The latter reference showed that the ALEE in (7) can be transformed to the following mixed Partial Differential Equation (PDE) for the Green's function variable, $\tilde{v}=\tilde{v}\left(y_{1}, U\right) \equiv \widetilde{c}^{2} \tilde{G}_{4}+\tilde{G}_{5}$ :

$$
\mathcal{L} \tilde{v}\left(y_{1}, U\right)=\mathcal{F}(\tilde{\boldsymbol{S}}),
$$

for $\epsilon=O(1)$ spreadrates in which the Favre-averaged speed of sound $\widetilde{c^{2}}$ satisfies the Crocco-Busemann relation for a heated flow (Eq. 17 and discussion below Eq. 16 in reference [5] or Crocco relation in an unheated flow that was used in [4]. The operator,

$$
\mathcal{L}\left(y_{1}, U\right) \equiv \widetilde{c^{2}} \frac{\partial}{\partial U} \frac{1}{\widetilde{c}^{2}} \tilde{D}_{0}+\tilde{X}_{1} \frac{\partial^{2}}{\partial U^{2}},
$$

is hyperbolic and

$$
\mathcal{F}(\tilde{\boldsymbol{S}})=\mathcal{F}\left(\tilde{S}_{1}, \tilde{S}_{r}, \tilde{S}_{5}\right):=\tilde{S}_{1}-\left(\frac{\tilde{S}_{5}}{\widetilde{c^{2}}}+D_{0} \tilde{S}_{r}\right) .
$$

where $\left(\tilde{S}_{1}, \tilde{S}_{r}, \tilde{S}_{5}\right)$ are defined through Eqs. (21), (14) and the line below (15) in reference [5]. The components, $\tilde{S}=\left\{\tilde{S}_{1}, \tilde{S}_{r}, \tilde{S}_{5}\right\}$, are functions of the Green's function components $\left(\tilde{G}_{1}, \tilde{G}_{r}\right)$ in 77 and mean flow field $\left(U, V_{r}\right)$ and the 


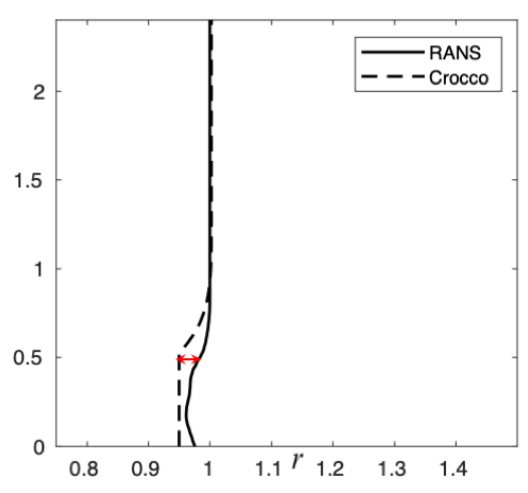

(a) $y_{1}=2$

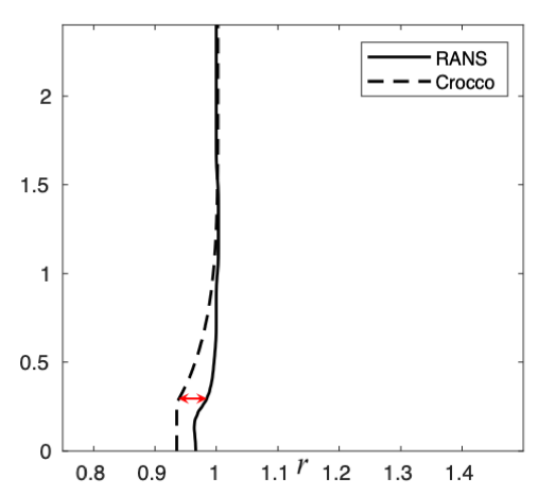

(c) $y_{1}=6$

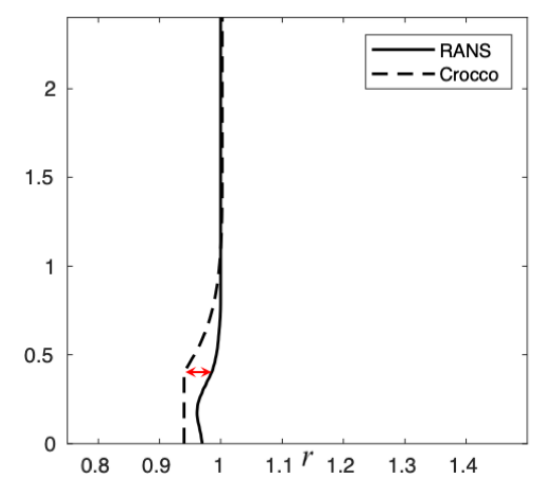

(b) $y_{1}=4$

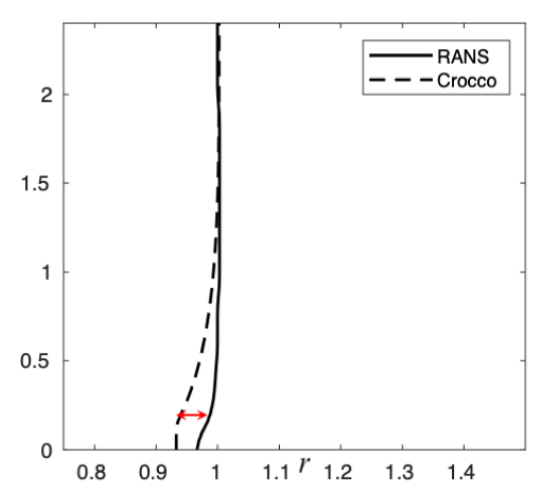

(d) $y_{1}=8$

Fig. 5 Comparison of the Crocco relation (Eq. 5.33 in [7]) against the RANS mean flow $\widetilde{c^{2}} / c_{\infty}^{2}$ for OP1.7 at various points in the jet.

streanwise/radial components of the mean flow advection vector $\left(\tilde{X}_{1}, \tilde{X}_{r}\right)$, determined by $(8)$. Note that the tilde on the Green's function indicates its functional space is now $\left(y_{1}, U\right)$ via the implicit function theorem [2].

In our case however, Fig. ?? shows that $\widetilde{c^{2}}$ departs significantly from that predicted by the Crocco-Busemann relation for OP1.3. The departure in Fig. ?? for OP1.7 is about 5\% relative to that given by the Crocco relation for the unheated single stream operating point, which is consistent with subsonic single stream jets analyzed in ASL19.

In order to accommodate this into the asymptotic solution of ALEE, we use a more general form of 9 , where $\widetilde{c^{2}}$ is taken to be a function of $U$, as $f\left(U\left(y_{1}, r\right)\right)$, where $f\left(U\left(y_{1}, r\right)\right.$ is now otherwise arbitrary. That is,

$$
\mathcal{L} \tilde{\mathcal{v}}\left(y_{1}, U\right)-\tilde{X}_{1}\left[(\gamma-1)+\frac{\partial^{2} \widetilde{c^{2}}}{\partial U^{2}}\right] \tilde{G}_{4}=\mathcal{F}(\tilde{\boldsymbol{S}}),
$$

(Eq. 16 in [5]) where $\mathcal{L}\left(y_{1}, U\right)$ is given by 10$]$.

\section{Asymptotic solution to $(12)$ in small jet spread rate limit}

When the temporal frequency is appropriately re-scaled as $\Omega=\omega / \epsilon=O(1)$ and considering the conditions across the surface $r=0$ in the $i=\phi$ component of (7]), the analysis in [5] shows that since the Green's function components, $\tilde{G}_{(r, \phi)}$, must remain bounded on the jet axis, $\tilde{G}_{(r, \phi)}=0$ at lowest order in $(7), 9$, , 11 . Hence, the right hand side of 9 , remains $O\left(\epsilon^{2}\right.$ (or at $O(1)$ relative to lowest order expansion) in the small jet spread rate limit $(\epsilon \ll O(1)-$ which an axisymmetric jet possess by definition). In other words, $\mathcal{F}(\boldsymbol{S})$ remains asymptotically sub-dominant in this limit. We 
summarise this next and show it leads to an asymptotic expansion of $\Gamma_{\lambda, j}$ that at its lowest order involves only a single term. We summarise this steps required to obtain this result next and show it leads to an asymptotic expansion of $\Gamma_{\lambda, j}$ that at its lowest order involves only a single term. The main difference to our previous papers is that the re-scaled solution to the variable, $\tilde{v}$ is now longer determined by a purely hyperbolic PDE. That is, the solution to $\bar{v}$ (defined below in 15 is now coupled with the solution to $\bar{G}_{4}$. This is discussed further below.

In order to proceed with the asymptotic analysis as defined in ASL19 we let all lengths be normalized by the $O(1)$ characteristic length scale, the nozzle diameter, $D_{J}$, and time scale $D_{J} / U_{J}$, where $U_{j}$ is the mean jet exit velocity. The fluid mechanical variables $(\tilde{\boldsymbol{v}}, p, \rho)$ may then be normalized by $U_{J}, \rho_{J} U_{J}^{2}$ and $\rho_{J}$.

Allowing the mean flow to vary over a slow streamwise length, $Y \equiv \epsilon y_{1}=O(1)$, corresponding to long streamwise length scales $y_{1}$, shows that it must expand according to (A.1-A.2) in G \& L:

$$
\tilde{v}_{i}=\left\{U(Y), V_{r}(Y, U)\right\}= \begin{cases}U+\epsilon U^{(1)}(Y, U)+O\left(\epsilon^{2}\right), & i=1 \\ \epsilon\left(V_{r}+\epsilon V_{r}^{(2)}\right)(Y, U)+O\left(\epsilon^{3}\right), & i=r\end{cases}
$$

when $\widetilde{c^{2}}=f(U)$ and remains arbitrary. We have not put superscripts on the lowest order mean flow components, that would otherwise appear as $\left(U^{(0)}, V_{r}^{(1)}\right)$ respectively; they will be taken as that computed by the RANS solution. Moreover at this order in $\epsilon: \bar{\rho}(Y, U)=\rho(U)$ and $\bar{p}(Y, U)=$ const. and the mean flow advection vector, $X_{i}(\boldsymbol{y})$, that enters in $\tilde{S}_{i}=\left\{\tilde{S}_{1}, \tilde{S}_{r}, \tilde{S}_{5}\right\}$, similarly expands as

$$
\tilde{X}_{i}=\left\{\tilde{X}_{1}, \tilde{X}_{r}\right\}(Y, U)= \begin{cases}\epsilon \bar{X}_{1}(Y, U)+\epsilon^{2} \tilde{X}_{1}^{(2)}(Y, U)+O\left(\epsilon^{3}\right), & i=1 \\ \epsilon^{2} \bar{X}_{r}^{(2)}(Y, U)+O\left(\epsilon^{3}\right), & i=r\end{cases}
$$

where the leading streamwise term, $\bar{X}_{1}^{(1)} \equiv \bar{X}_{1}=V_{r}(\partial U / \partial r)$ and $\bar{X}_{r}^{(2)}=\left(U \partial / \partial Y+V_{r} \partial / \partial r\right) V_{r}$. Hence, measured from the jet centerline, the mean flow separates into an inner region, given by (13) \& (14), where (inner) radial co-ordinate $r=O(1)$, and an outer region where this expansion break downs; i.e., at large radial locations (with respect to inner variable, $r$ ) for which $R \equiv \epsilon r=O(1)$. Allowing $g_{v 4}^{a}(\boldsymbol{y}, \tau \mid \boldsymbol{x}, t)$ to depend on time, $\tau$, through the $O(1)$ slowly breathing time $\tilde{T}=\epsilon \tau$ allows mean flow non-parallelism to enter the lowest order asymptotic expansion of $\tilde{v}$ everywhere in the flow (and not just in the critical layer at supersonic speeds as in G\&L's solution) and at $M a=O(1)$.

The scaled Fourier transform:

$$
\begin{aligned}
\tilde{v}(Y, U) & \equiv \frac{\epsilon}{4 \pi|x|} e^{i \Omega X / c_{\infty}} \bar{v}\left(Y, U|X,| x_{T} \mid, 0 ; \Omega\right) \\
& =\frac{1}{2 \pi \epsilon} \int_{-\infty}^{\infty} e^{i \Omega\left(\tilde{T}_{0}-\tilde{T}\right)}\left(\widetilde{c}^{2} \tilde{g}_{44}+\tilde{g}_{54}\right)\left(Y, U|X,| x_{T} \mid, 0 ; \tilde{T}_{0}-\tilde{T}\right) d\left(\tilde{T}_{0}-\tilde{T}\right),
\end{aligned}
$$

that is now determined by the homogeneous form of $[12$ at arbitrary $\Omega=\omega / \epsilon=O(1)$ frequencies when $\mathcal{F}(\tilde{\boldsymbol{S}})=o(1)$; i.e.,

$$
\overline{\mathcal{L}} \bar{v}(Y, U) \equiv \widetilde{c^{2}} \frac{\partial}{\partial U}\left(\frac{1}{\widetilde{c}^{2}} \bar{D}_{0} \bar{v}\right)-\bar{X}_{1} \frac{\partial^{2} \bar{v}}{\partial U^{2}}-\tilde{X}_{1}\left[(\gamma-1)+\frac{\partial^{2} \widetilde{c}^{2}}{\partial U^{2}}\right] \bar{G}_{4}=0, \text { for } \epsilon \ll O(1),
$$

where by the implicit function theorem, $\bar{v}\left(y_{1}, r\right) \equiv \bar{v}(Y, U) \equiv \widetilde{c}^{2} \bar{G}_{4}+\bar{G}_{5}$ is related to the zeroth-order azimuthal mode through the inverse Fourier transform of azimuthal expansion of $\tilde{v}$ and $\bar{G}_{4}$ in $(\Phi-\phi)$ (see Eq. 2.20 \& 2.21 in ASL19) where $\left(X, T_{0}\right)=\epsilon\left(x_{1}, t\right)$ are appropriate $O(1)$ slow variables at $\left(x_{1}, t\right)$. by

Matching $v(Y, U)$ to the inner limit of the outer solution using Van Dyke's rule shows that it is uniquely determined

$$
\begin{gathered}
\bar{v}(Y, 0) \rightarrow-i \Omega c_{\infty}^{2} e^{-i \Omega Y \cos \theta / c_{\infty}} \\
\frac{\partial \bar{v}}{\partial U}(Y, 0) \rightarrow-i \Omega c_{\infty} \cos \theta e^{-i \Omega Y \cos \theta / c_{\infty}}
\end{gathered}
$$

on the non-characteristic curve $U=0$, with $Y \geq 0$ (where, as indicated above, $U \rightarrow 0$ corresponds to outer limit, $r \rightarrow \infty$. Note that the bypass stream is localized within $O\left(D_{J}\right)$ distances from the jet center line such that the flow outside of the jet (i.e. from both streams) is zero and the matching conditions are given by (17) \& (18). The coefficient $\bar{X}_{1}$ is the streamwise component of the mean flow advection vector (equation 5.15 in [7]) and $\bar{D}_{0}=i \Omega+U \partial / \partial Y$. Note 
the re-scaling of Fourier transform of $g_{v 4}^{a}(\boldsymbol{y}, \tau \mid \boldsymbol{x}, t)$ via a relation of the form of $(15)$ above for the composite solution $\bar{v}=\widetilde{c}^{2} \bar{G}_{4}+\bar{G}_{5}$ determined by $(16)$ or propagator 21$]$ below that depends on $\left.\left(\bar{G}_{1}, \bar{G}_{4}\right), \bar{G}_{5}\right)$, is only necessary inasmuch as it simplifies matching conditions (17) \& (18).

In order to solve 16 we require another equation relating $\bar{v}$ and $\bar{G}_{4}$. This takes the form of

$$
\bar{D}_{0} \bar{v}=\widetilde{c^{2}} D_{0} \bar{G}_{4}
$$

when, commensurate with the asymptotic arguments discussed above, the right hand side term in Eq. (15) of [5] is set to zero. The operator, $D_{0}$, is given by $D_{0}=\bar{D}_{0}+\bar{X}_{1} \partial / \partial U$ with $\bar{D}_{0}$ defined above. The matching conditions on $\bar{G}_{4}$ follow easily using 17$]$ \& 18 since $\bar{v} \rightarrow \bar{G}_{4}$ in the outer wave equation region.

A. Propagator solution at $\omega / \epsilon=O(1)$ and $\epsilon \ll O(1)$

Afsar et al. [5] show that for supersonic heated jets using the momentum flux term alone (i.e. taking $n_{2}=n_{3}=0$ in (41) in [5] such that temperature associated correlation functions are negligible) gives accurate predictions up to $S t \sim 0.7-0.8$. This approximation was also confirmed by Gryazev et al. in [6]. Hence introducing this approximation shows that low frequency form of the spectrum, $I(\boldsymbol{x}, \boldsymbol{y} ; \omega)$, reduces to

$$
I(\boldsymbol{x}, \boldsymbol{y} ; \omega) \rightarrow\left(\frac{\epsilon}{c_{\infty}^{2}|x|}\right)^{2}\left|G_{12}\right|^{2} \Phi_{1212}^{*}
$$

The Fourier transformed adjoint Green's functions $\left(\bar{G}_{1}, \bar{G}_{4}\right)$ represent the Green's function for the streamwise linearized momentum and energy equations of the generalized acoustic analogy ([2] \& [5]); they enter (??) through the propagator components:

$$
G_{12}=\tilde{G}_{12}(Y, U)=\frac{\partial \tilde{G}_{1}}{\partial r}-(\gamma-1) \tilde{G}_{4} \frac{\partial U}{\partial r}+O(\epsilon)
$$

\section{Green's function analysis}

\section{A. Effect of square brackets on the solution to $\bar{v}$ in 16 )}

While the Crocco relation holds very well in Fig 5 for the OP1.7 condition, there is a large disparity between the LES mean flow and the Crocco-Busemann relation for the heated set point, OP1.3. Recall that Afsar et al.[5] found that the latter relation, assumed to valid for a heated flow, allows the Green's function problem to be given by the solution to the Hyperbolic form of (16) when the square brackets are identically zero. We compared the solution to the Green's function problem using the mean flow for the OP1.3 set point and found that, remarkably, there is very little difference in the solutions to the Green's function problem when the square brackets in 16 is retained or not. We show this in Fig. 6 below.

In the what remains of the paper we will calculate $\bar{v}$ for both operating conditions by solving 116 when the square brackets in the latter equation are assumed to be negligible and (16) does not require the use of an auxiliary equation 19]) because of the coupling between the primary variable of the low frequency Green's function problem, $\bar{v}$, and it primitive dependency $\hat{G}_{4}$. (16) then de-couples and reduces to the solution of the Hyperbolic PDE for $\bar{v}$ and is subject to (17) \& (18). This is now the same basic equation as given in [7] for isothermal flows and which was shown to remain valid in heated flows in [4].

\section{B. Convergence of the hyperbolic form of 16 ,}

In Fig. 7 we perform a grid convergence study to show that the solution to $\bar{v}(Y, U)$ and $\partial \bar{v}(Y, U) / \partial U$ is converged. The latter is needed in the extraction of the streamwise component of the adjoint vector Green's function for the linearized momentum equation (Eq. 5.27 in [7]). We compute these solutions for OP1.3 and OP1.7 at the peak noise location, $\theta=30^{\circ}$. We use 'grid-4' (550 $\times 400$ points) to compute the propagator term in 20 ) and the acoustic spectrum predictions. 


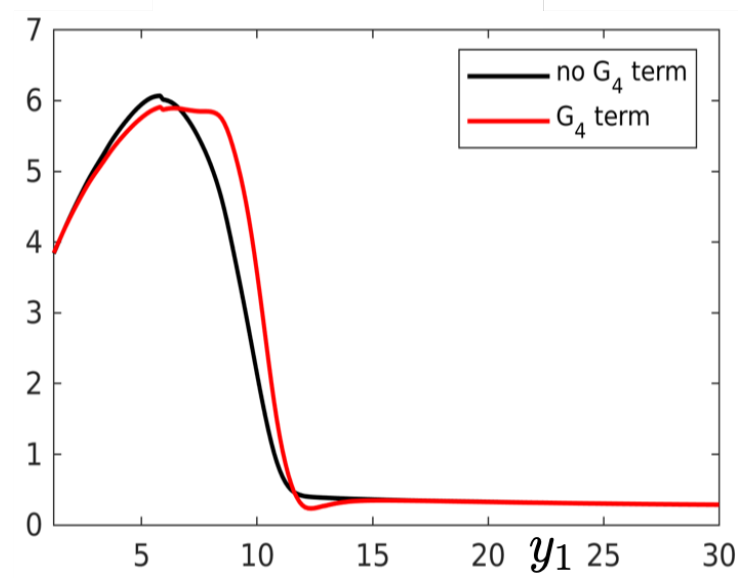

(a) $S t=0.01$

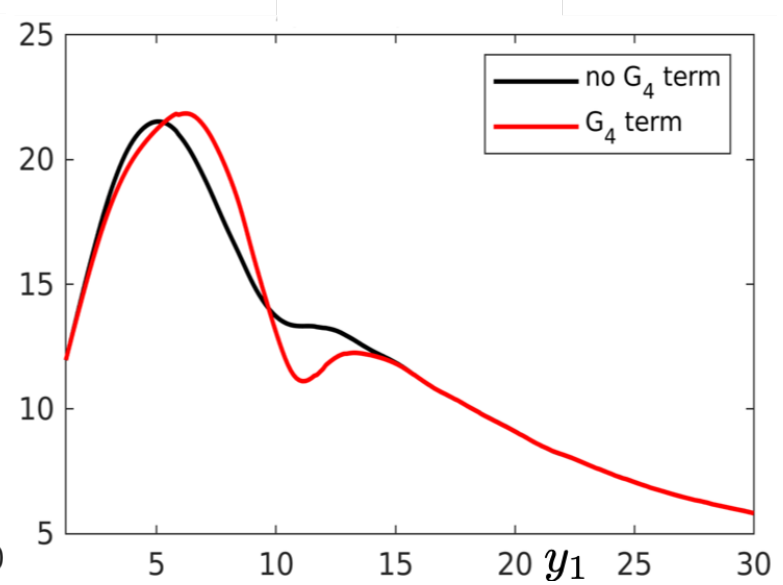

(b) $S t=0.1$

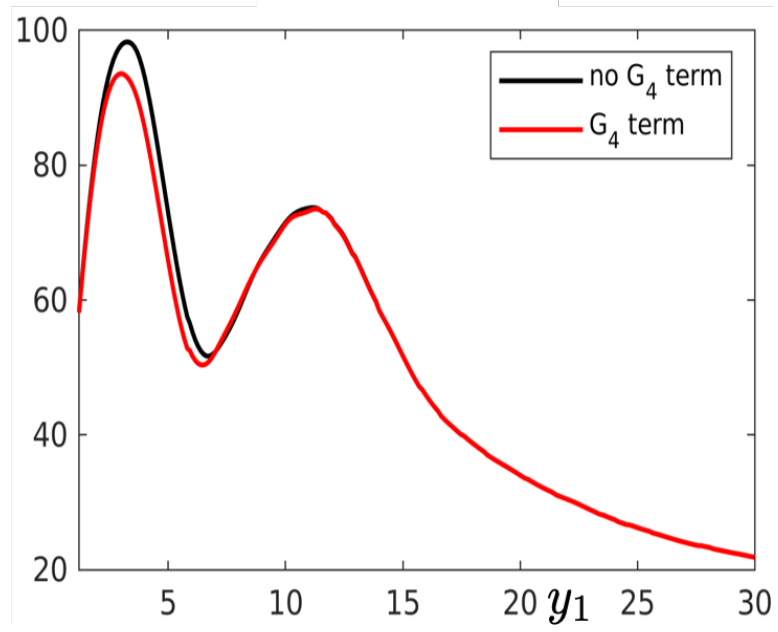

(c) $S t=0.5$

Fig. 6 Solution to $\bar{v}$ using $(16)$ and 19 subject to $\left(17,, 18\right.$, and similar conditions on $\bar{G}_{4}$ at $\theta=30^{\circ}$ for OP1.3.

\section{Determination of $\Phi_{1212}^{*}\left(\boldsymbol{y}, k_{1}, k_{T}^{2}, \omega\right)$ in 20}

\section{A. The model parameters for $R_{1212}$}

The spectral tensor component, $\Phi_{1212}^{*}\left(y, k_{1}, k_{T}^{2}, \omega\right)$, is explicitly related to $R_{1212}$ via the space-time Fourier transform:

$$
\Phi_{1212}^{*}(\boldsymbol{y}, \boldsymbol{k} ; \omega)=\frac{1}{2 \pi} \int_{V_{\infty}(\boldsymbol{\eta})} \int_{-\infty}^{\infty} e^{i(\boldsymbol{k} \cdot \boldsymbol{\eta}-\omega \tau)} R_{1212}\left(\boldsymbol{y}, \eta_{1}, \eta_{T}, \tau\right) d \tau d \boldsymbol{\eta},
$$

We model $R_{1212}\left(\boldsymbol{y}, \eta_{1}, \eta_{T}, \tau\right)$ in the same manner as [5] (their Eq. 43) whereby its normalized auto-correlation, $R_{1212}(\boldsymbol{y}, \tau) / R_{1212}(\boldsymbol{y})$, takes the simple exponential form $\sim\left(1-a_{1} \bar{\tau} l_{r}\right) e^{-l_{r} \bar{\tau}}$ at zero spatial separations, $\boldsymbol{\eta}=0$ where $\bar{\tau}$ is the appropriately normalized time delay. In Fig. 8, we compare the latter form of $R_{1212}(y, \tau) / R_{1212}(y)$ to the LES data for the OP1.3 and 1.7 set points at the core and bypass aerodynamic radii at the streamwise location at the end of the jet potential core. That is, at $x \sim 6$; note that while this length reduces with jet heating for OP1.3 the contour plots in Fig. 5 a shows that the reduction is small compared to Fig. $2 a$ - the normalization of the spatial field point is with nozzle radius, $r_{J}$ here. The hand-tuned values of the turbulence length scale ratio, $l_{r}=l_{1} / l_{0}$, and the anti-correlation parameter, $a_{1}$, is given in Table 2 obtained by the comparisons shown in Fig. 8. 


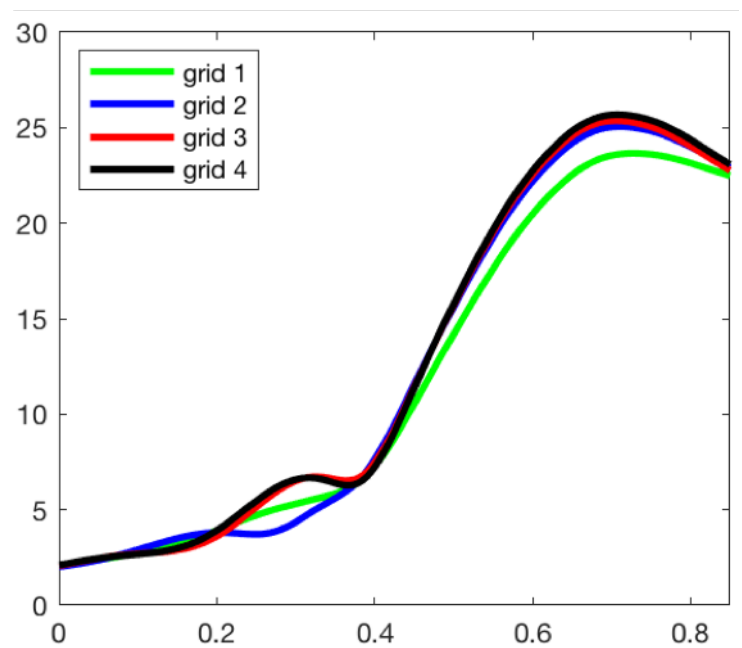

(a) OP1.3: $\bar{v}(Y, U)$

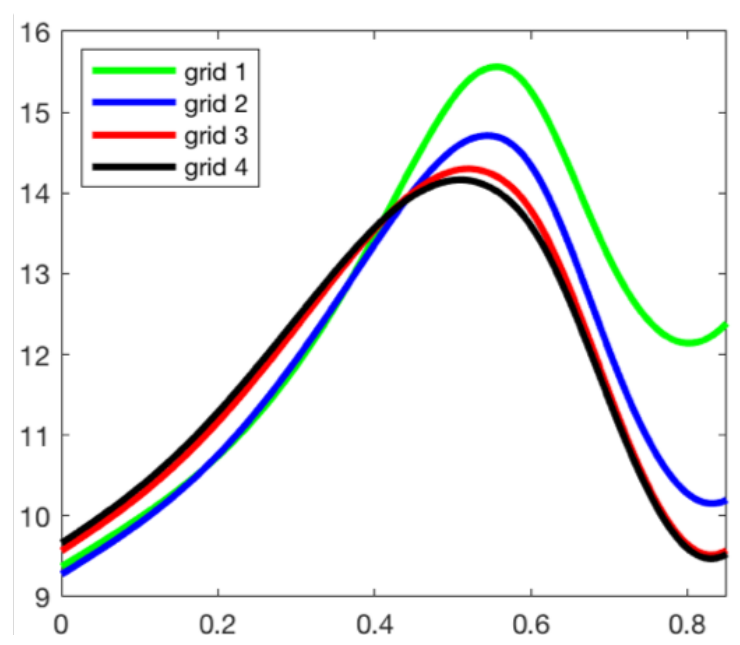

(c) OP1.7: $\bar{v}(Y, u)$

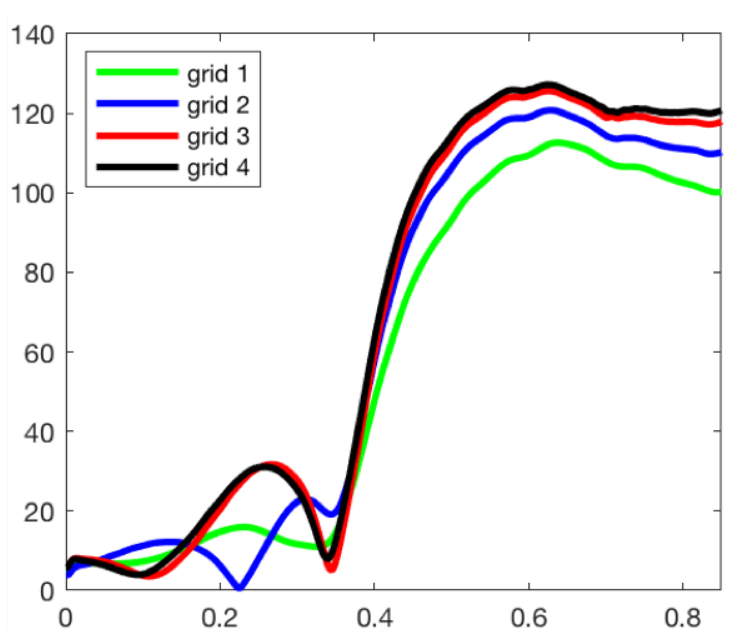

(b) OP1.3: $\partial \bar{v}(Y, U) / \partial U$

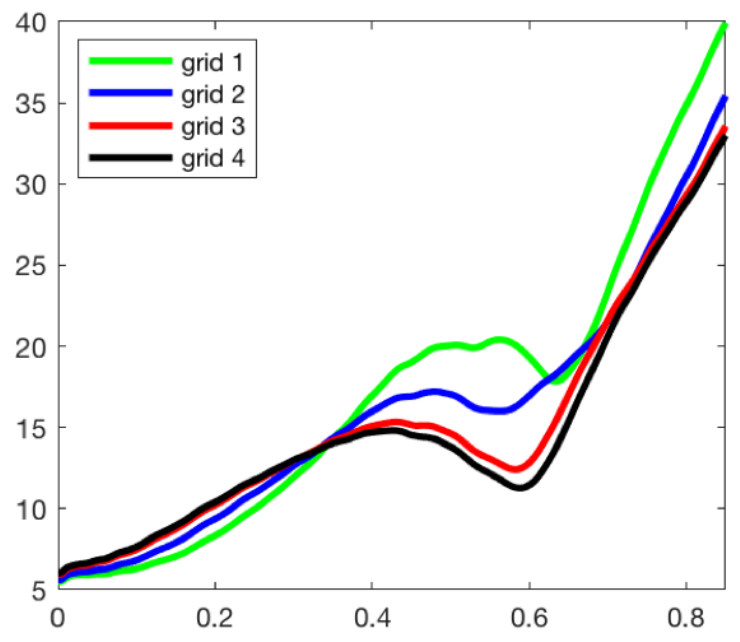

(d) OP1.7: $\partial \bar{v}(Y, U) / \partial U$

Fig. 7 Convergence of $\bar{v}$ and $\partial \bar{v} / \partial U$ in the solution to the hyperbolic form of 16 .

As well as determining the $\left(l_{r}=l_{1} / l_{0}, a_{1}\right)$ parameters, the amplitude of the correlation function can be found by scaling it with respect to the density-weighted turbulence kinetic energy (TKE, $\rho k$ ) fields obtained from the LES mean flow field. In other words, $R_{1212}(\boldsymbol{y}, \mathbf{0} ; 0)=a_{1212} \bar{\rho}^{2}(\boldsymbol{y}) k^{2}(\boldsymbol{y})$ As indicated in [5], $a_{1212}$ could also be a function of $\boldsymbol{y}$ but is often approximated by a single value because it is usually relatively constant[9]. In Fig. (9) we show the ratio $a_{1212} \sim R_{1212}(\boldsymbol{y}, \mathbf{0} ; 0) / \bar{\rho}^{2}(\boldsymbol{y}) k^{2}(\boldsymbol{y})$ is more-or-less equal to 0.25 for both operating points in the bypass aerodynamic radius and at the end of the potential core.

\section{Jet noise predictions}

In Figs. (10) \& (11) we show the $\theta=\left(30^{\circ}, 40^{\circ}\right)$ for OP1.3 and OP1.7. Fig. (10) uses only the non-parallel flow Green's function that is determined by the numerical solution to 16 (with the square brackets in the latter equation set to zero) and subject to $(17) \&(18)$. The predictions are seen to remain accurate (i.e. well within $0.5 \mathrm{~dB}$ of the acoustic data up to a Strouhal number of $S t \sim 0.4-0.5$ at $\theta=30^{\circ}$ depending on the operating point. Following reference [4], we show in Fig. (11) that indeed the prediction range can be extended to cover the full frequency band of $S t=[0.01,1.0]$ when the parallel flow solution to $(16)$ is used additively with the non-parallel flow solution at frequencies beyond the peak frequency of $S t=0.5$ and acts as a form of 'composite asymptotic solutionf 4 . This approach, postulated by (3.1) in [4], uses the plane wave solution to 16 for $\bar{v}$ that is $\bar{v} \sim A(U ; \Omega) \exp ^{-i \Omega Y \cos \theta / c_{\infty}}$ (Eq. 7.1 in [7]) where $A(U ; \Omega)$ 


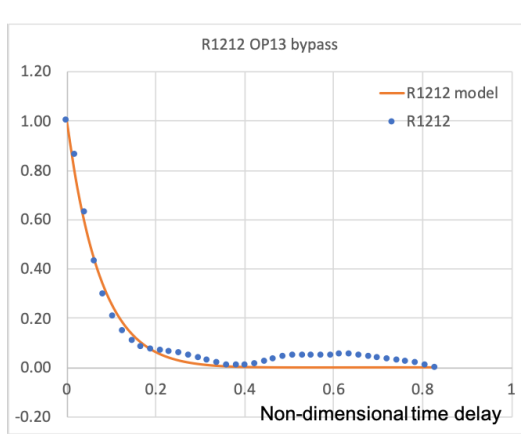

(a) OP1.3 bypass

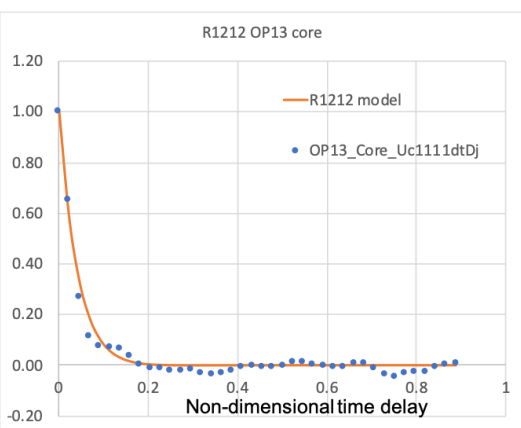

(b) OP1.3 core

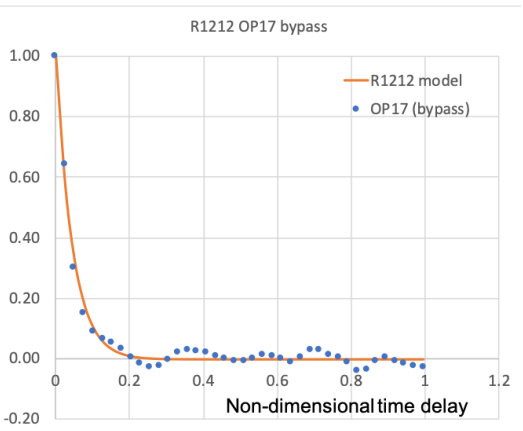

(c) OP1.7 bypass

Fig. 8 Comparison of $R_{1212}(y, \tau) / R_{1212}(y)$ in [5] (Eq. 43) to LES data.

$\begin{array}{lll}\text { Operating Point }(\mathrm{OP}) & l_{r}=l_{1} / l_{0} & a_{1} \\ \text { OP1.3 Core } & 20 & 0.2 \\ \text { OP1.3 Bypass } & 11 & 0.2 \\ \text { OP1.7 Bypass } & 20 & 0.1\end{array}$

Table 2 Turbulence parameters for $R_{1212}(y, \tau)$ in 22

is the appropriate amplitude pre-factor of the locally parallel flow solution to 16 when $\bar{X}_{1}$ is set to zero in the latter hyperbolic equation.

\section{Conclusion}

In this paper we have developed the analytical machinery to solve the small-spread rate asymptotic form of the ALEE, (7) for complex dual axisymmetric stream jets in which the outer bypass stream interacts with the core stream flow. The theory we have developed in the form of (16) and (19) extend our previous analyses by allowing the mean flow speed of sound to be any $f\left(U\left(y_{1}, r\right)\right.$ where $f$ is otherwise arbitrary. This was necessary because RANS simulations of the dual stream jet a supersonic heated conditions (see Fig. 4) indicated that $\widetilde{c^{2}}$ radically departs from the Crocco-Busemann form that was assumed in our previous work (see reference [5]) on a single stream flow. However, the Green's function calculations in Fig. 5 shows the extended form of the the non-parallel flow asymptotic theory for the adjoint pressure-like Green's function given by (16) is not too sensitive to the square brackets in that Partial differential equation that captures the effect of the Crocco-Busemann relation not being valid. (The square brackets would be identically zero in (16) if either the Crocco or Crocco-Busemann relation were found to valid).

The predictions in Figs. (10) and (11) show that excellent agreement is found with this approach for observations angles in the vicinity of the peak radiated noise. While this agreement with our predicted spectrum using (20) remains very close to the acoustic data, typically up to frequencies whereby the Strouhal number, $S t \sim 0.4-0.5$ depending upon the operating point, the agreement reduces at the observation angle, $\theta$, increases (i.e. at $\theta=40^{\circ}$ ). See Fig. 10 . Thus exemplifies the 'peak-noise' applicability of the asymptotic theory first developed in [7] and later extended to heated flows by [5]. The prediction range can be naturally extended to almost the full Strouhal number range (Fig. 11), however, if use is made of the parallel flow solution to the adjoint Green's function equation (16), at frequencies beyond the peak. This is utilized in the form of a type of composite asymptotic solution for the primary Green's function variable in the problem (this is defined as $\bar{v}$ in 16, 17] and 18) and is given by Eq. (3.1) in [4].

\section{Acknowledgments}

Computational resources from HPC2, Mississippi State University, are appreciated. MZA would like to thank Strathclyde University for financial support from the Chancellor's Fellowship.. 


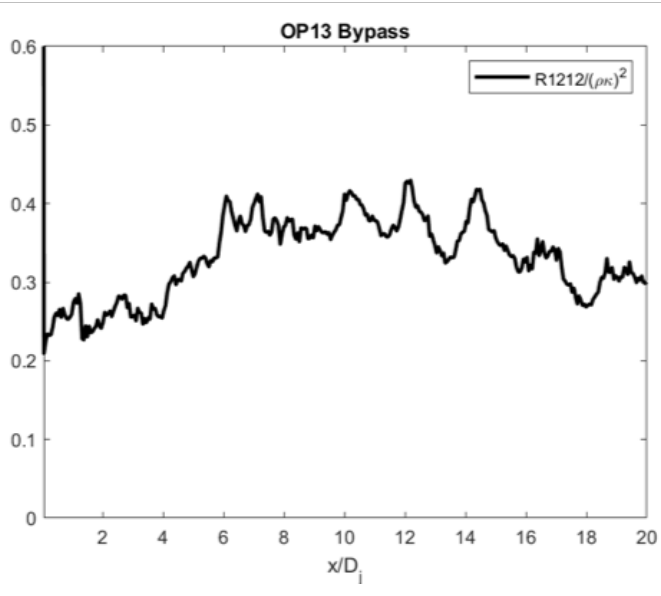

(a) OP1.3 bypass

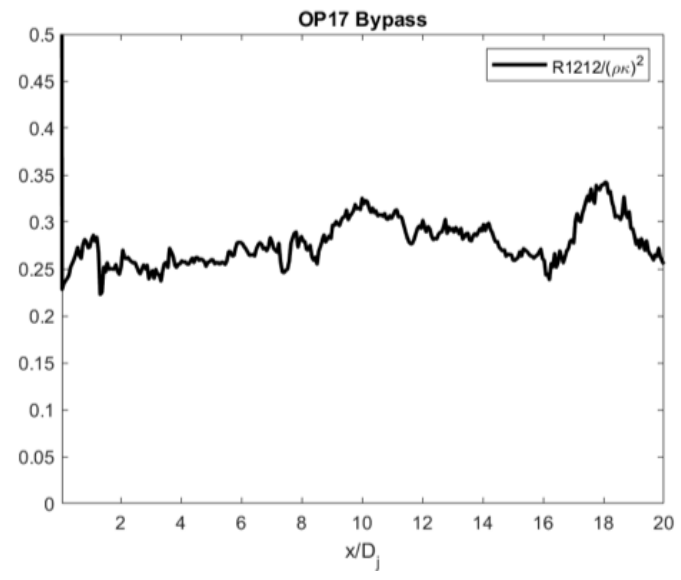

(b) OP1.7 bypass

Fig. 9 Relative constancy of the pre-factor $a_{1212}$ in the proportionality relation, $R_{1212}(\boldsymbol{y}, \mathbf{0} ; 0)=a_{1212} \bar{\rho}^{2}(\boldsymbol{y}) k^{2}(\boldsymbol{y})$.

\section{References}

[1] Lighthill, M. J., “On sound generated aerodynamically: I. General theory,” Proc. R. Soc. Lon., A, Vol. 211, 1952 , p. 564.

[2] Goldstein, M. E., “A generalized acoustic analogy,” J. Fluid Mech., Vol. 488, 2003, p. 315.

[3] Monin, A. S., and Yaglom, A. M., Statistical fluid mechanics, vol II, chapter and pages.

[4] Afsar, M. Z., Sescu, A., and Leib, S. J., "Modelling and prediction of the peak-radiated sound in subsonic axisymmetric air jets using acoustic analogy-based asymptotic analysis," Phil. Trans. R. Soc. A, Vol. 377, 2019.

[5] Afsar, M. Z., Sescu, A., and Sassanis, V. G., "Effect of non-parallel mean flow on the acoustic spectrum of heated supersonic jets: Explanation of "jet quietening"," Phys. Fluids, Vol. 31, 105107, 2019.

[6] Gryazev, V., Markesteijn, A. P., and Karabasov, S. A., "Low-Order models of dual-stream jet noise with temperature effects based on the Goldstein generalised acoustic analogy," 25th AIAA Aeroacoustics Conference, 2019.

[7] Goldstein, M. E., Sescu, A., and Afsar, M. Z., "Effect of non-parallel mean flow on the Green's function for predicting the low-frequency sound from turbulent air jets," J. Fluid Mech., Vol. 695, 2012, p. 199.

[8] Goldstein, M. E., and Leib, S. J., “The Aero-acoustics of slowly diverging supersonic jets,” J. Fluid Mech., Vol. 488, 2008 , p. 315.

[9] Karabasov, S. A., Afsar, M. Z., Hynes, T. P., Dowling, A. P., McMullan, W. A., Pokora, C. D., Page, G. J., and McGuirk, J. J., "Jet noise: acoustic analogy informed by Large-Eddy Simulation," AIAA J., Vol. 48, 2010, p. 1312. 


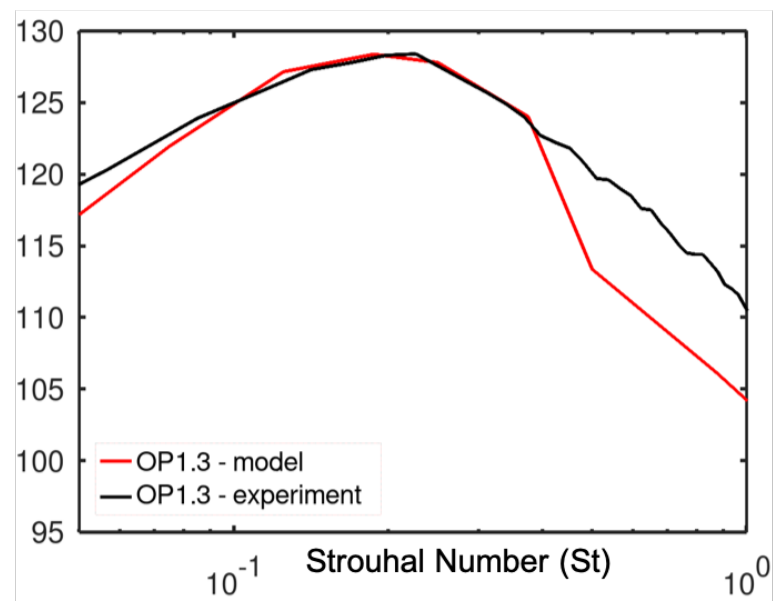

(a) OP1.3: $\theta=30^{\circ}$

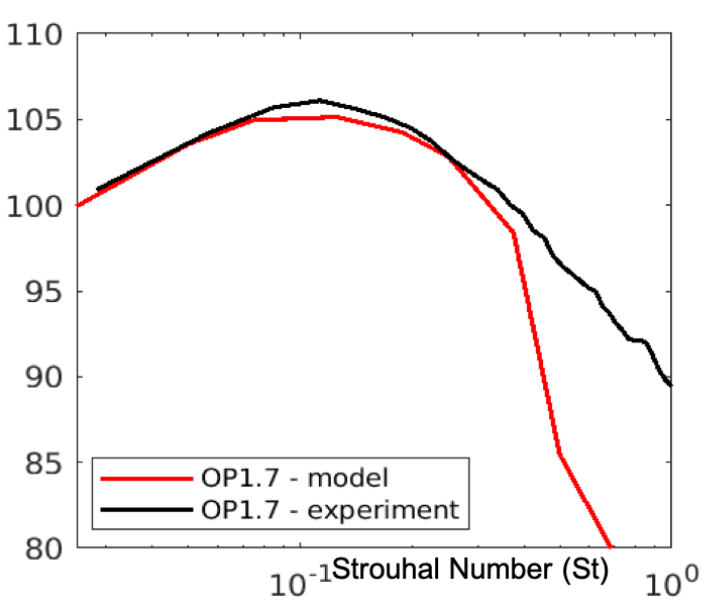

(c) OP1.7: $\theta=30^{\circ}$

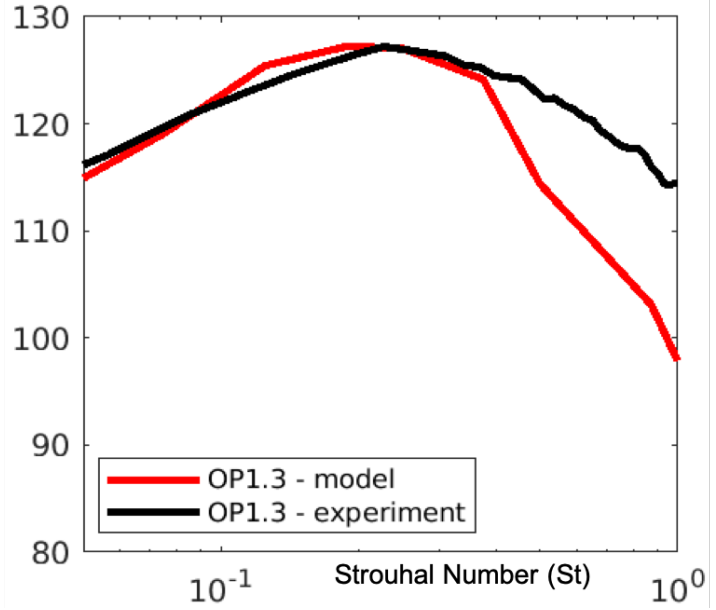

(b) OP1.3: $\theta=40^{\circ}$

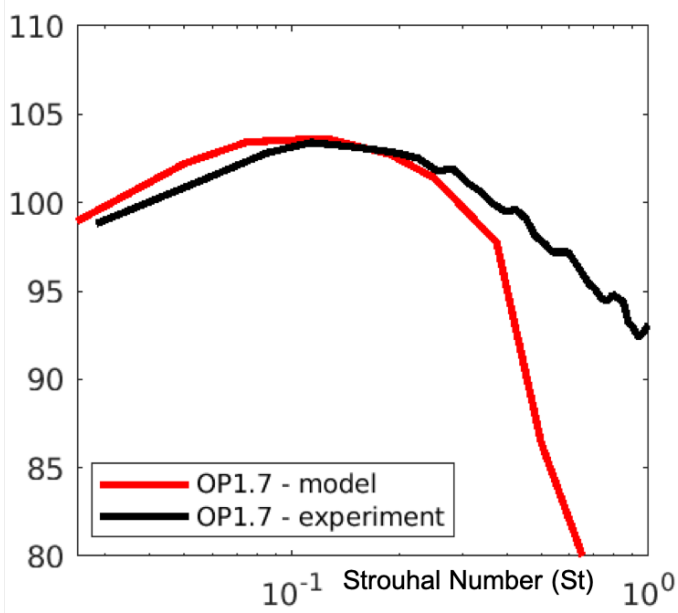

(d) OP1.7: $\theta=40^{\circ}$

Fig. 10 SPL computed via $S P L=10 \log _{10} 4 \pi\left(\rho U_{J}^{2}\right)^{2} I(\boldsymbol{x} ; \omega) / P_{r e f}^{2}$ where $P_{r e f}=2 \times 10^{-5}$ Pa. The spectrum $I(x ; \omega)$ is determined by integrating (20) over $\boldsymbol{y}=\left(y_{1}, r\right)$. 


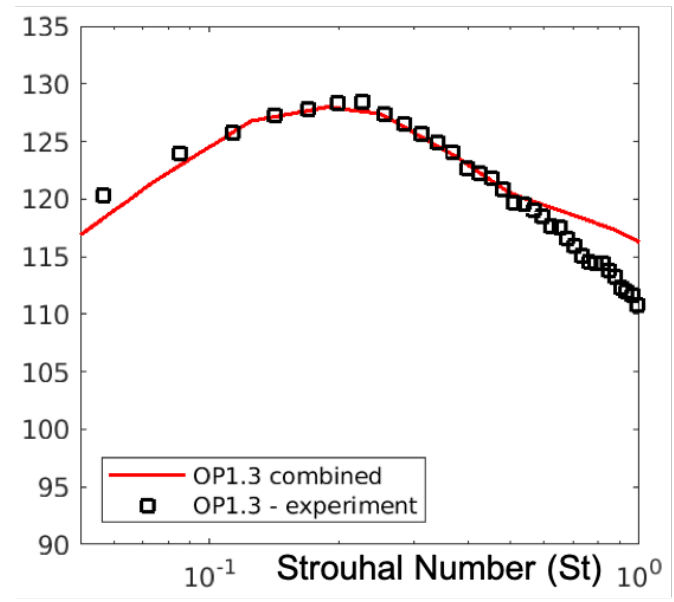

(a) OP1.3: $\theta=30^{\circ}$

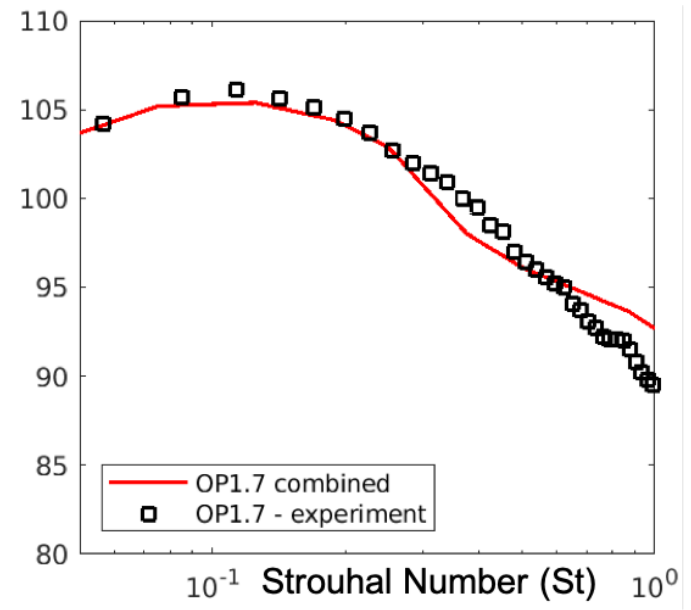

(c) OP1.7: $\theta=30^{\circ}$

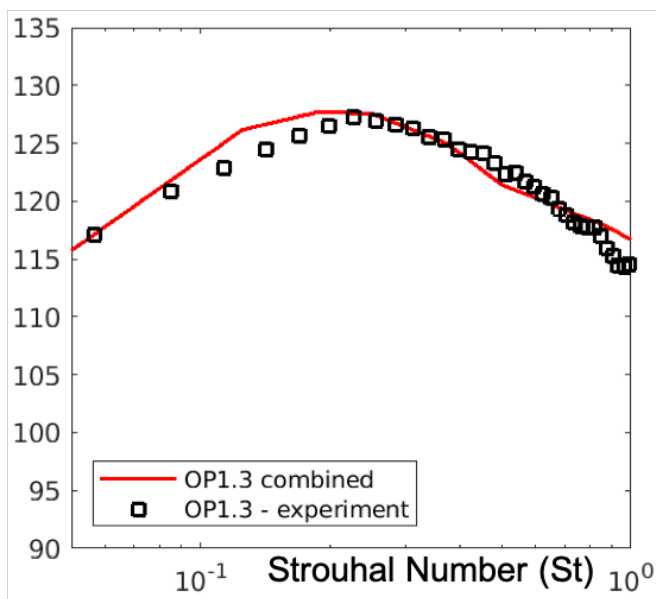

(b) OP1.3: $\theta=40^{\circ}$

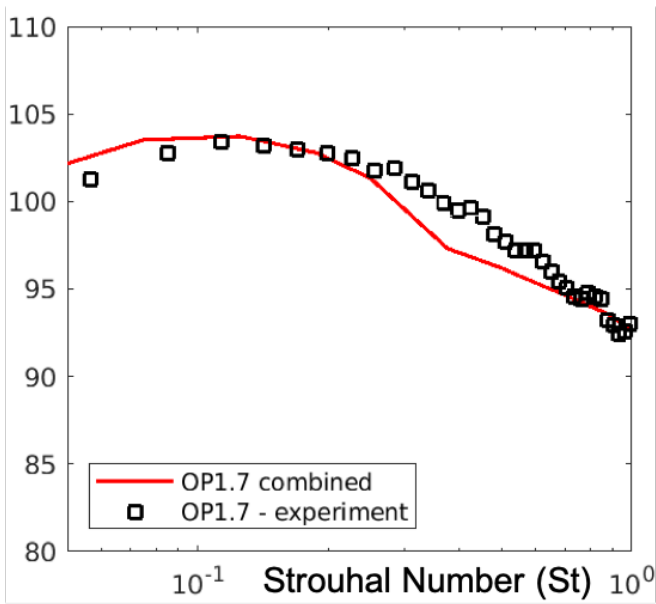

(d) OP1.7: $\theta=40^{\circ}$

Fig. 11 SPL prediction against acoustic data in [6]. SPL computed via $S P L=10 \log _{10} 4 \pi\left(\rho U_{J}^{2}\right)^{2} I(x ; \omega) / P_{r e f}^{2}$ where $P_{r e f}=2 \times 10^{-5} \mathrm{~Pa}$. The spectrum $I(\boldsymbol{x} ; \omega)$ is determined by integrating (20) over $\boldsymbol{y}=\left(y_{1}, r\right)$. 